'TRIFLE' CENTRE

FOR APPLIED MATHEMATICS IN NATURAL SCIENCE

Self-scaling tumor growth

Jürgen Schmiegel

Research Report

No. 07 I August 2005 


\section{Self-scaling tumor growth}

This Thiele Research Report is also Research Report number 460 in the Stochastics Series at Department of Mathematical Sciences, University of Aarhus, Denmark. 



\title{
Self-scaling tumor growth
}

\author{
Jürgen Schmiegel \\ Thiele Centre for Applied Mathematics in Natural Sciences, \\ Department of Mathematical Sciences, \\ Aarhus University, DK-8000 Aarhus, Denmark, *
}

\begin{abstract}
We study the statistical properties of the star-shaped approximation of in vitro tumor profiles. The emphasis is on the two-point correlation structure of the radii of the tumor as a function of time and angle. In particular, we show that spatial two-point correlators follow a cosine law. Furthermore, we observe self-scaling behaviour of two-point correlators of different orders, i.e. correlators of a given order are a power law of the correlators of some other order. This power-law dependence is similar to what has been observed for the statistics of the energydissipation in a turbulent flow. Based on this similarity, we provide a Lévy based model that captures the correlation structure of the radii of the star-shaped tumor profiles.
\end{abstract}

PACS: 87.18.Hf, 89.75.Da, 02.50.Ey

KEYWORDS: Lévy bases, growth models, tumor growth, self scaling, correlators

\section{Introduction}

The medical term cancer refers to a wide variety of different tumor species, each with specific characteristics. A realistic modelling framework for tumor growth may be a valuable tool, not only for understanding the underlying dynamics, but also as a support for grading the malignancy of tumor tissue. The interest of the problem has led to the formulation of numerous growth models $[1,2,3,4]$ (and references therein).

There are several works where the fractal dimension of tumors has been measured with the aim to classify and determine their malignant nature $[2,5]$. The tumor boundary has a fractal nature, and its morphology can be characterized by a fractal dimension. The fractal dimension is closely related to the roughness of the surface. In general, the roughness of the interface between the tumor and the non-tumor region is an indicator of whether the tumor is likely to become infiltrative or not. Tumors whose interfaces are very rough are seen to be more aggressive [5].

The fractal dimension is a very rough characteristic of the complexity of tumor profiles. Apparently very different profiles have the same fractal dimension (in particular,

${ }^{*}$ The author is much indebted to A. Brú and co-workers for allowing to use their data sets. This work was supported by the Carlsberg Foundation. 
any smooth profile has the fractal dimension 1). In this paper, we propose the use of correlators as a measure of complexity that characterizes the dynamics of tumor profiles in more detail. In this approach, a growing tumor is modelled as a stochastic process in space and time. The description of a tumor as a stochastic process is justified by the adaptability of biological systems to a changing environment, which in turn implies their sensitivity to local variations of many dynamically relevant parameters. In a complex systems like a growing tumor local conditions may be expected to change randomly with time. Thus stochastic effects are always present [6].

To account for the statistical properties of tumor profiles we propose a modelling framework that is based on the integration with respect to a homogeneous Lévy basis. A similar approach proved useful for the modelling of the turbulent energy dissipation field $[7,8]$. The strength of the Lévy based modelling framework lies in the fact that it allows to analytically control the correlation structure of the model in accordance with experimental findings.

In Section 2 we briefly describe the type of data we use for our statistical analysis. In particular we introduce the description of an in vitro growing tumor profile as a star-shaped object. Correlators are introduced in Section 3 and it is shown that spatial correlators of tumor profiles follow a cosine law. In addition, spatial correlators have the property of self-scaling, i.e. correlators of a given order are a power law of correlators of some other order. These striking statistical properties of a growing tumor are the basis for the formulation of the modelling framework which is presented in Section 4 . Section 5 shows some results from numerical simulations that illustrate the potential of the proposed model in generating typical tumor profiles. Section 6 concludes.

\begin{tabular}{c|cccccc} 
& $A$ & $T$ & $t_{0}$ & $a$ & $h$ & $\phi_{0}$ \\
\hline $\mathrm{t}=21$ & -0.021 & 21 & 19 & 0.04 & -0.033 & 0.19 \\
$\mathrm{t}=25$ & -0.019 & 25 & 17 & 0.02 & -0.033 & 0.19 \\
$\mathrm{t}=55$ & -0.021 & 18 & 4 & 0.01 & -0.067 & 0.23
\end{tabular}

Table 1: Parameters $A$ (in units [radiants] $]^{-1}$ ), $T$ and $t_{0}$ (in units of the finite step size $\Delta t$ ), $a$ and $h$ (in units $[\Delta t]^{-1}[\text { radiants }]^{-1}$ where $\Delta t$ is the finite step size) for the simulation of the star-shaped radii $r_{t}(\phi)$ at times $t=21,25,55$ (in units of the finite step size $\Delta t$ ). The parameters for the Gaussian background field $Z$ are $\mu=0.1$ and $\sigma^{2}=1$.

\section{Description of the data}

The data we use for our statistical analysis of tumor profiles are snapshots of a growing brain tumor in vitro. The details of the set-up of such in vitro experiments may be found in [2]. The experimental conditions allow cells to grow mainly on a plate surface, thus the tumor can be considered a two-dimensional system. Here we are mainly interested in the boundary of the growing tumor. We approximate this boundary as a star-shaped profile with radial function

$$
R_{t}(\phi)=\max \left\{R: \mathbf{c}_{0}+R \mathbf{e}_{\phi} \in Y_{t}\right\}
$$

where $Y_{t}$ denotes the two-dimensional domain occupied by the tumor at time $t, \mathbf{c}_{\mathbf{0}}$ denotes the centre of mass of the tumor at time $t=0$ and $\mathbf{e}_{\phi}$ is the unit vector in direction $\phi \in[0,2 \pi]$. 

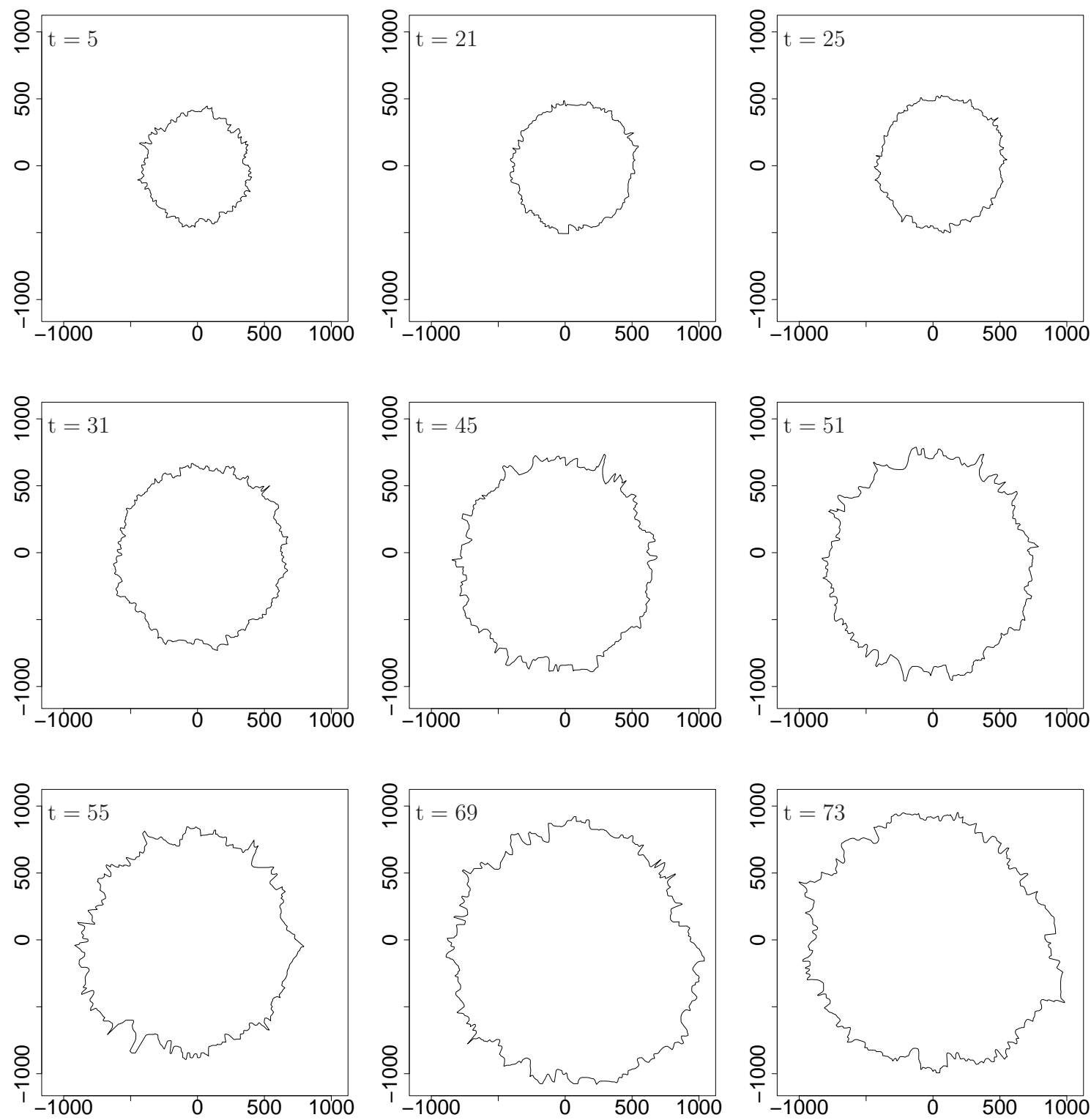

Figure 1: Star-shaped approximation of the tumor (in microns) at times $t=$ $5,21,25,31,45,51,55,69,73$ (in hours).

Figure 1 shows an example of such star-shaped profiles at various times $t$, originally analyzed in [2]. We observe structures at very different scales with strongly localized outbursts of different size. Due to the unrestricted growth of the tumor in in vitro experiments we can expect the profiles to be statistically isotropic. A comparison of these star-shaped profiles with the original profiles as observed in the experiment [2] shows that (1) approximates the growing tumor to a high accuracy. For the star-shaped approximation, we neglect details of the tumor profiles where small regions of non-tumor tissue are surrounded by tumor cells.

For the stochastic modelling of profiles we normalize the radial function

$$
r_{t}(\phi) \equiv \frac{R_{t}(\phi)}{\mathrm{E}\left\{R_{t}(\phi)\right\}}
$$

where $\mathrm{E}\{\}$ denotes the expectation and $\mathrm{E}\left\{R_{t}(\phi)\right\}$ is the mean radius at time $t$, assumed to be independent of $\phi$. Thus, $\mathrm{E}\left\{r_{t}(\phi)\right\}=1$ for all times $t$. For the estimation of 
expectations, we perform spatial averaging. Because of the normalization, we focus on the development of the shape of the tumor.

\section{Spatial correlators}

The profiles in Figure 1 reveal structures at very different scales. A complete description of the multi-scale structure of the tumor at time $t$ requires, in principle, the knowledge of all mixed moments of the form

$$
\mathrm{E}\left\{r_{t}\left(\phi_{1}\right) \cdot \ldots \cdot r_{t}\left(\phi_{n}\right)\right\}, \quad 1 \leq n \leq N
$$

where $2 \pi / N$ denotes the angular resolution of the profiles. Here, we restrict the analysis of the spatial variation of $r_{t}(\phi)$ to two-point correlators of order $\left(n_{1}, n_{2}\right)$

$$
c_{n_{1}, n_{2}}(t, \Delta \phi) \equiv \frac{\mathrm{E}\left\{r_{t}(\phi)^{n_{1}} r_{t}(\phi+\Delta \phi)^{n_{2}}\right\}}{\mathrm{E}\left\{r_{t}(\phi)^{n_{1}}\right\} \mathrm{E}\left\{r_{t}(\phi+\Delta \phi)^{n_{2}}\right\}} .
$$

It is to note that the spatial correlators $c_{n_{1}, n_{2}}(t, \Delta \phi)$ do not depend on $\phi$ for statistically isotropic profiles.

\subsection{The cosine law}

Figure 2 shows the logarithm of the estimated spatial correlators $c_{n_{1}, n_{2}}(t, \Delta \phi)$ with $\left(n_{1}, n_{2}\right)=(1,1)$ at various times $t$ with $0 \leq \Delta \phi \leq \pi$. Spatial correlators are monotonically decreasing on $[0, \pi]$ with $c_{1,1}(\pi / 2)=1$ for most of the times that are displayed in Figure 2. One can distinguish two different regimes for the behaviour of spatial correlators. For not too small angular distances $\Delta \phi>\phi_{0}(t)$ and most of the times $t$ it is reasonable to fit a cosine behaviour (shown as the solid line in Figure 2)

$$
\log \left(c_{n_{1}, n_{2}}(t, \Delta \phi)\right)=b_{n_{1}, n_{2}}(t) \cos (\Delta \phi), \quad \Delta \phi>\phi_{0}(t)
$$

with a time dependent amplitude $b_{n_{1}, n_{2}}(t)$. For the small angular distances $\Delta \phi<\phi_{0}(t)$ we observe deviations from the cosine behaviour (5). Similar results hold for the higher order spatial correlators (see Figure 4(a)). Irrespective of the order $\left(n_{1}, n_{2}\right)$, spatial correlators $c_{n_{1}, n_{2}}(t, \Delta \phi)$ follow the cosine law (5). In the following we refer to the angle $\phi_{0}(t)$, that marks the validity of the cosine behaviour (5), as the critical angle. The property of self-scaling of correlators in Section 3.2 then shows that $\phi_{0}(t)$ does not depend on the order $\left(n_{1}, n_{2}\right)$.

The estimated amplitudes $b_{1,1}(t)$ used in Figure 2 are displayed in Figure 3 as a function of time $t$. For the times $t=5,31,73$ the spatial correlators in Figure 2 show a considerable scatter which results in the lowest three values of the estimated amplitudes $b_{1,1}(t)$. For the remaining times, there is no clear time dependence of $b_{1,1}(t)$.

The cosine behaviour (5) for $\Delta \phi>\phi_{0}(t)$ can be rewritten, using the definition (4), as

$$
\mathrm{E}\left\{r_{t}(\phi)^{n_{1}} r_{t}(\phi+\Delta \phi)^{n_{2}}\right\}=A_{n_{1}, n_{2}}(t) \exp \left\{\frac{l_{n_{1}, n_{2}}\left(r_{t}(\phi), r_{t}(\phi+\Delta \phi)\right)}{L_{n_{1}, n_{2}}(t)}\right\}
$$

with a time dependent amplitude $A_{n_{1}, n_{2}}(t)=\mathrm{E}\left\{r_{t}^{n_{1}}\right\} \mathrm{E}\left\{r_{t}^{n_{2}}\right\}$, a time dependent scale $L_{n_{1}, n_{2}}(t)=\mathrm{E}\left\{r_{t}^{n_{1}}\right\} \mathrm{E}\left\{r_{t}^{n_{2}}\right\} / b_{n_{1}, n_{2}}(t)$ and a time dependent distance function

$$
l_{n_{1}, n_{2}}\left(r_{t}(\phi), r_{t}(\phi+\Delta \phi)\right)=\left\langle\mathrm{E}\left\{r_{t}^{n_{1}}\right\} \mathbf{e}_{\phi}, \mathrm{E}\left\{r_{t}^{n_{2}}\right\} \mathbf{e}_{\phi+\Delta \phi}\right\rangle
$$



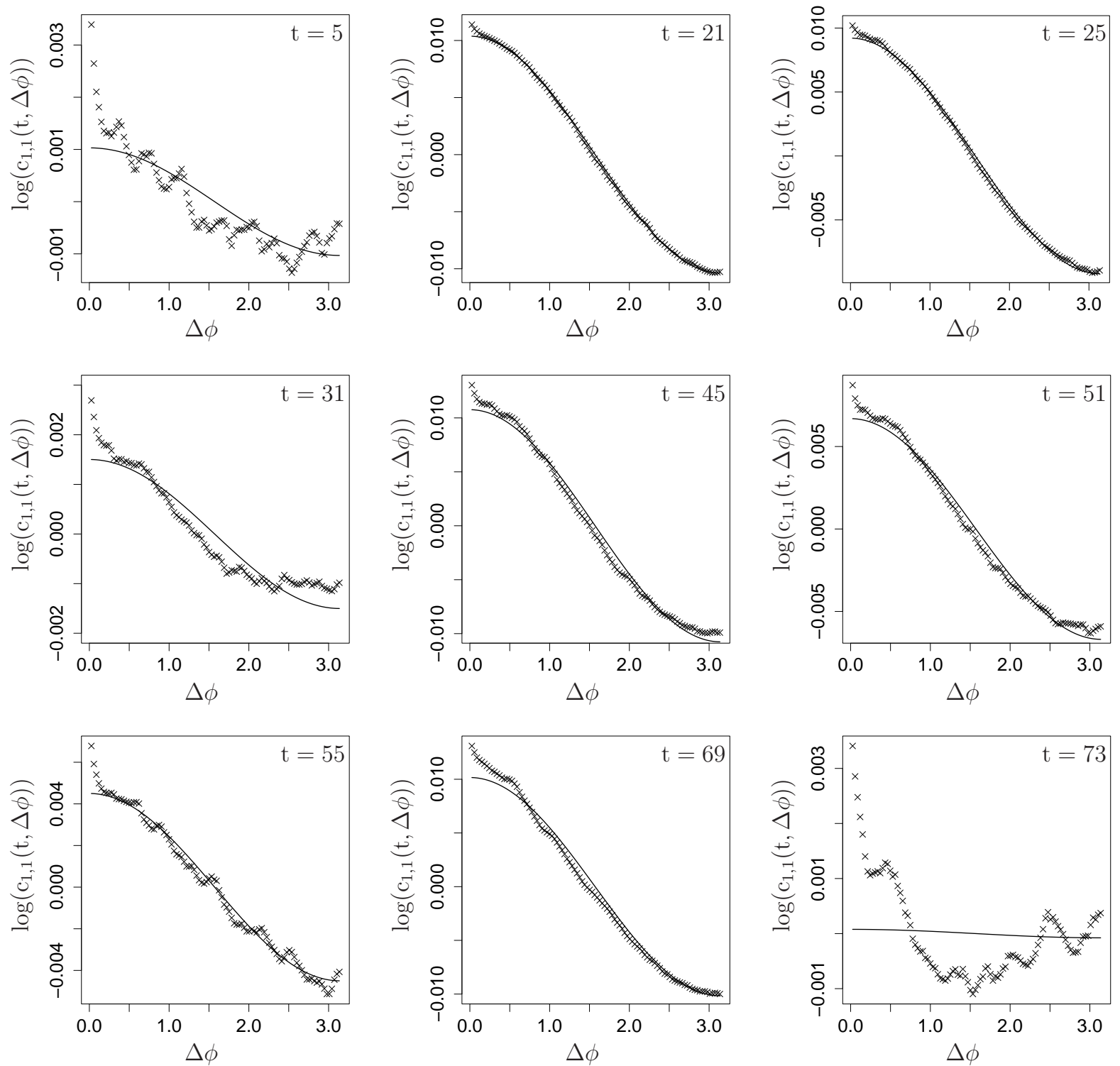

Figure 2: Logarithm of spatial correlators $c_{1,1}(t, \Delta \phi)$ as a function of $\Delta \phi$ (radiants) of the star-shaped tumor at times $t=5,21,25,31,45,51,55,69,73$ (in hours). The solid lines are of the form $b_{1,1}(t) \cos (\Delta \phi)$.

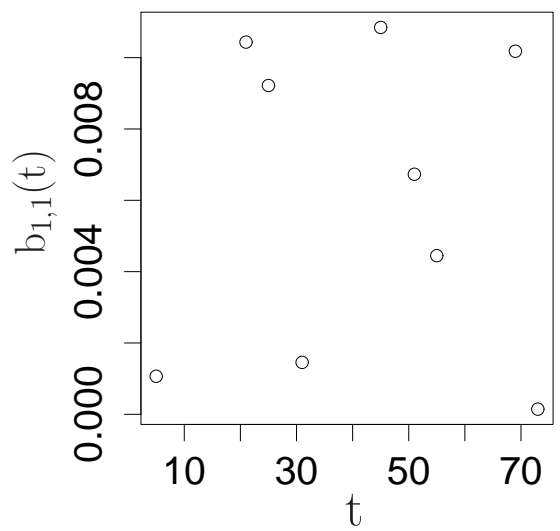

Figure 3: Estimated amplitude $b_{1,1}(t)$ at times $t=5,21,25,31,45,51,55,69,73$ (in hours). 
where \langle\rangle denotes the Euclidean scalar product. Here we use the abbreviation $\mathrm{E}\left\{r_{t}^{n_{1}}\right\}=$ $\mathrm{E}\left\{r_{t}(\phi)^{n_{1}}\right\}$ for statistically isotropic profiles. Written in the form (6), the cosine behaviour (5) is equivalent to exponential decay where the correlation is caused by the projection of $\mathrm{E}\left\{r_{t}^{n_{1}}\right\} \mathbf{e}_{\phi}$ on $\mathrm{E}\left\{r_{t}^{n_{2}}\right\} \mathbf{e}_{\phi+\Delta \phi}$.

\subsection{Self-scaling of correlators}

The structure of (5) as a product of an order-dependent factor $b_{n_{1}, n_{2}}(t)$ and an angledependent factor $\cos (\Delta \phi)$ immediately implies that, for $\Delta \phi>\phi_{0}(t)$, correlators of a given order $\left(n_{1}, n_{2}\right)$ are a power law of correlators of some other order $\left(m_{1}, m_{2}\right)$

$$
c_{n_{1}, n_{2}}(t, \Delta \phi)=\left(c_{m_{1}, m_{2}}(t, \Delta \phi)\right)^{k_{t}\left[m_{1}, m_{2} ; n_{1}, n_{2}\right]} .
$$

This property is called self-scaling of correlators. The self-scaling exponents $k_{t}\left[m_{1}, m_{2} ; n_{1}, n_{2}\right]$ are independent of $\Delta \phi$ and given by

$$
k_{t}\left[m_{1}, m_{2} ; n_{1}, n_{2}\right]=\frac{b_{n_{1}, n_{2}}(t)}{b_{m_{1}, m_{2}}(t)} .
$$

Figure 4 confirms the self-scaling relation (8) for $t=55$. We clearly observe that plotting $\log \left(c_{n_{1}, n_{2}}(t, \Delta \phi)\right)$ as a function of $\log \left(c_{m_{1}, m_{2}}(t, \Delta \phi)\right)$ results in a straight line through the origin with constant slopes $k_{t}\left[m_{1}, m_{2} ; n_{1}, n_{2}\right]$. Similar results hold for all times $t$ that are displayed in Figure 2 .

It is important to note that the self-scaling relation (8) holds for all angular distances $\Delta \phi$, including the deviations from the cosine behaviour (5) below the critical angle $\phi_{0}(t)$. Therefore, we may expect the deviations from (5) at small angular distances to be of the form

$$
\log \left(c_{n_{1}, n_{2}}(t, \Delta \phi)\right)-b_{n_{1}, n_{2}}(t) \cos (\Delta \phi)=d_{n_{1}, n_{2}}(t) f_{t}(\Delta \phi), \quad \Delta \phi<\phi_{0}(t),
$$

where the amplitude $d_{n_{1}, n_{2}}(t)$ does not depend on $\Delta \phi$ and the function $f_{t}(\Delta \phi)$ does not depend on the order $\left(n_{1}, n_{2}\right)$. Furthermore, the validity of (8) below the critical angle implies $\phi_{0}(t)$ being independent of the order $\left(n_{1}, n_{2}\right)$ (see Figure $4(\mathrm{a})$ ). For the small scale amplitudes $d_{n_{1}, n_{2}}(t)$ we get, using (8)-(10),

$$
\frac{d_{n_{1}, n_{2}}(t)}{d_{m_{1}, m_{2}}(t)}=\frac{b_{n_{1}, n_{2}}(t)}{b_{m_{1}, m_{2}}(t)}=k_{t}\left[m_{1}, m_{2} ; n_{1}, n_{2}\right] .
$$

Figure 5 shows the estimated self-scaling exponents $k_{t}\left[m_{1}, m_{2} ; n_{1}, n_{2}\right]$ as a function of time $t$. The self-scaling exponents can be assumed to be independent of time $t$ for all orders $\left(n_{1}, n_{2}\right)$ and $\left(m_{1}, m_{2}\right)$ that are displayed in Figure 5. Furthermore, we get to high accuracy

$$
\frac{\ln \left(c_{n_{1}, n_{2}}(t, \Delta \phi)\right)}{\ln \left(c_{m_{1}, m_{2}}(t, \Delta \phi)\right)}=k_{t}\left[m_{1}, m_{2} ; n_{1}, n_{2}\right]=\frac{n_{1} n_{2}}{m_{1} m_{2}} .
$$

This simple expression for the self-scaling exponents is the basic motivation for modelling the radius process $r_{t}(\phi)$ as the exponential of a Gaussian process in Section 4.2.

Combining (12) with (11) implies the factorization

$$
b_{n_{1}, n_{2}}(t)=b_{n_{1}, n_{2}} B(t)
$$

and

$$
d_{n_{1}, n_{2}}(t)=d_{n_{1}, n_{2}} D(t)
$$

into order-dependent factors $b_{n_{1}, n_{2}}=d_{n_{1}, n_{2}}=n_{1} n_{2}$ and time-dependent amplitudes $B(t)$ and $D(t)$, respectively. 

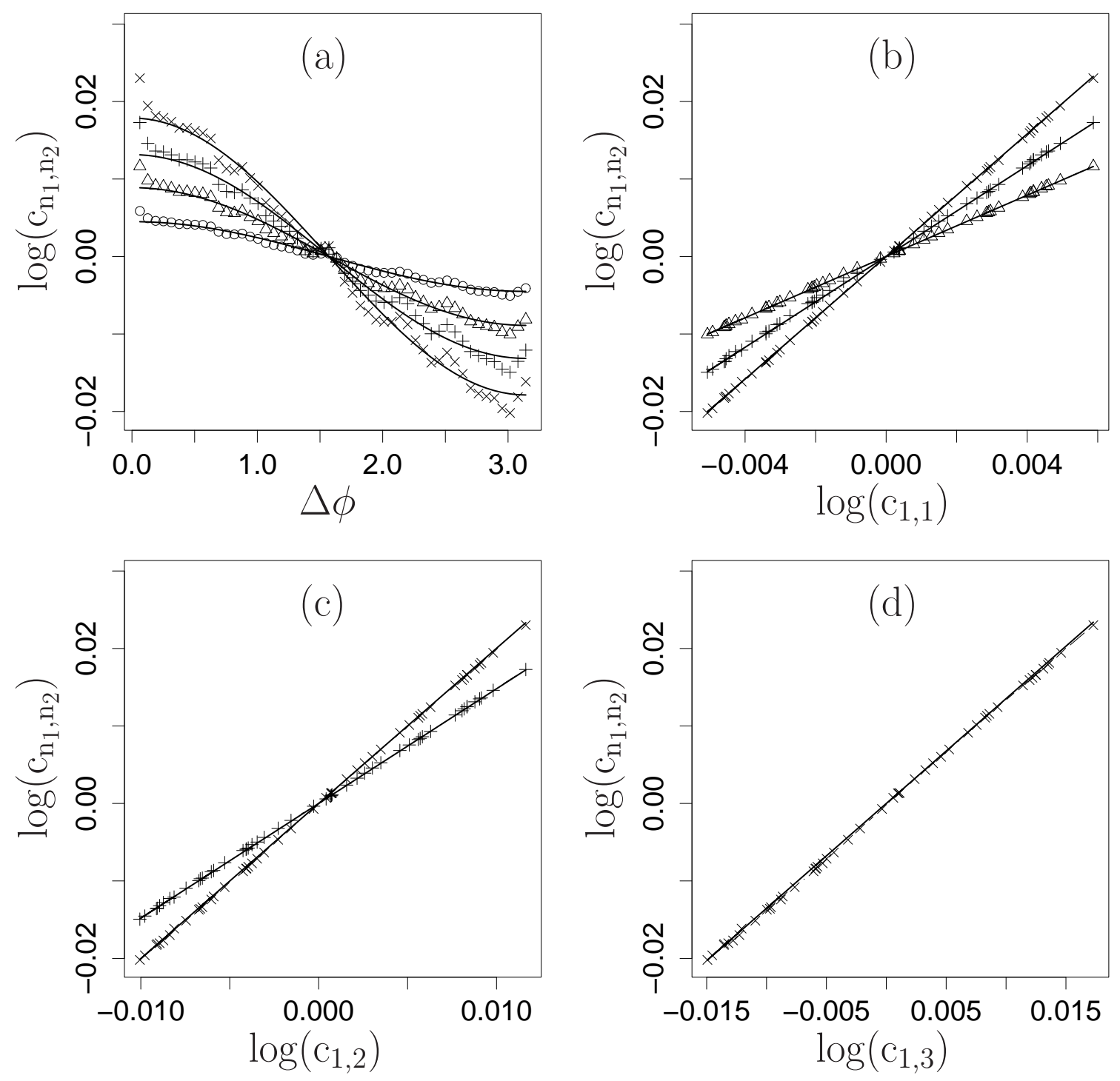

Figure 4: (a) Logarithm of spatial correlators $c_{n_{1}, n_{2}}(t, \Delta \phi)$ of orders $\left(n_{1}, n_{2}\right)=(1,1)(\mathrm{o})$, $\left(n_{1}, n_{2}\right)=(1,2)(\triangle),\left(n_{1}, n_{2}\right)=(1,3)(+)$ and $\left(n_{1}, n_{2}\right)=(2,2)(\times)$ at time $t=55$ (in hours) as a function of $\Delta \phi$ (radiants). The solid lines are of the form $b_{n_{1}, n_{2}}(t) \cos (\Delta \phi)$. (b) Logarithm of spatial correlators $c_{n_{1}, n_{2}}(t, \Delta \phi)$ as a function of $\log c_{1,1}(t, \Delta \phi)$ for $\left(n_{1}, n_{2}\right)=(1,2),(1,3),(2,2)$. The solid lines are of the form $k_{t}\left[1,1 ; n_{1}, n_{2}\right] \log c_{1,1}(t, \Delta \phi)$. (c) Logarithm of spatial correlators $c_{n_{1}, n_{2}}(t, \Delta \phi)$ as a function of $\log c_{1,2}(t, \Delta \phi)$ for $\left(n_{1}, n_{2}\right)=(1,3),(2,2)$. The solid lines are of the form $k_{t}\left[1,2 ; n_{1}, n_{2}\right] \log c_{1,2}(t, \Delta \phi)$. (d) Logarithm of spatial correlators $c_{2,2}(t, \Delta \phi)$ as a function of $\log c_{1,3}(t, \Delta \phi)$. The solid line is of the form $k_{t}\left[1,3 ; n_{1}, n_{2}\right] \log c_{1,3}(t, \Delta \phi)$. 

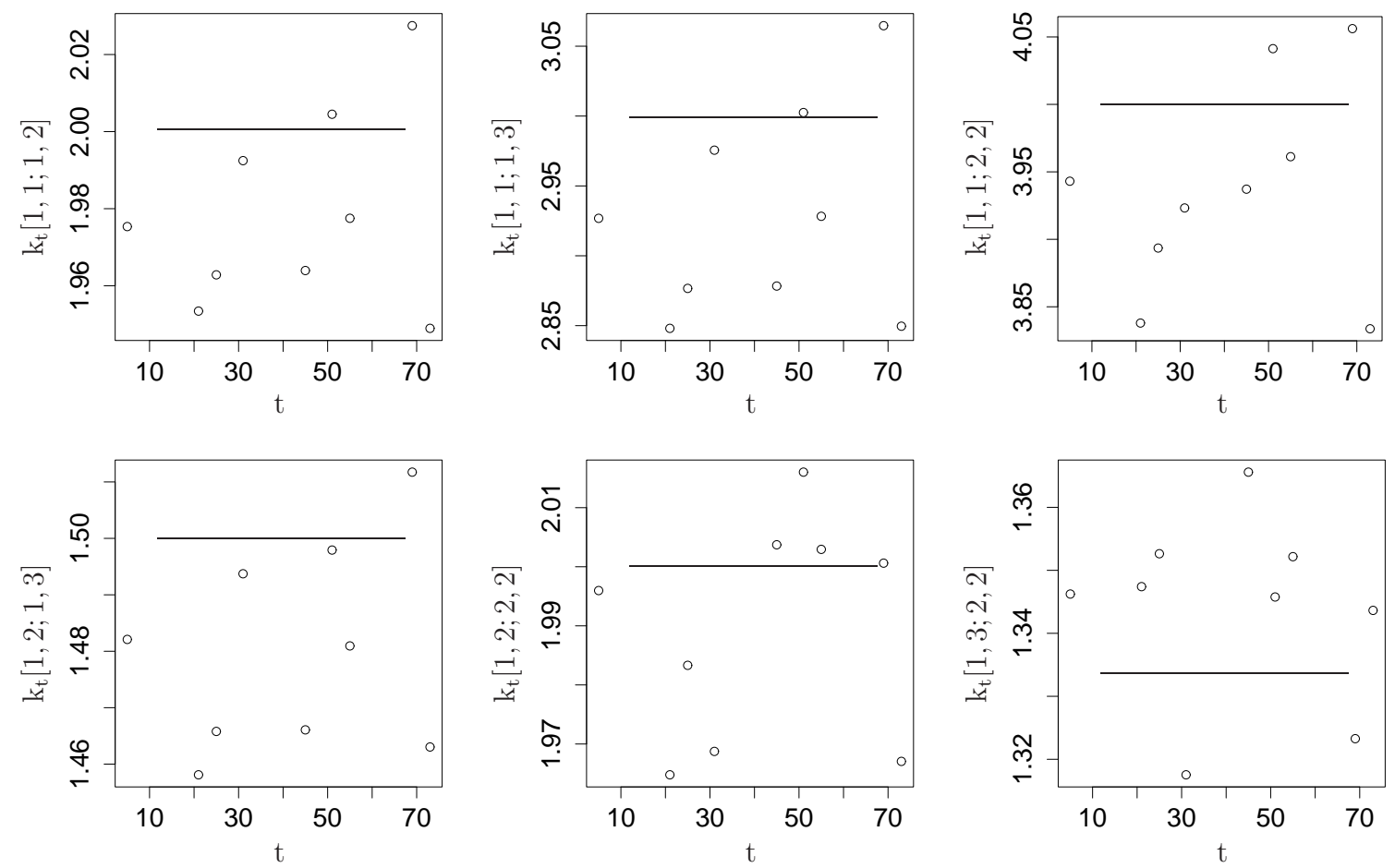

Figure 5: Self-scaling exponents $k_{t}\left[m_{1}, m_{2} ; n_{1}, n_{2}\right]$ as a function of time $t$ (in hours). The solid lines denote the values $\left(n_{1} n_{2}\right) /\left(m_{1} m_{2}\right)$.

\section{A Lévy based modelling framework}

The self scaling property (8) is also observed for the statistics of the energy dissipation in turbulent flows [8]. Moreover, the dynamics of the energy dissipation in turbulent flows reveal a multi-scale structure with strongly localized bursts that are similar, at least qualitatively, with the corresponding strong fluctuations of the tumor profiles. In the turbulence context, a general modelling framework that accounts for the strong variability of fluctuations and the self-scaling behaviour of correlators is based on the integration with respect to Lévy bases $[7,8]$. Thus, it is natural to apply this Lévy based modelling framework also to the dynamics of the tumor profiles.

\subsection{The general modelling framework}

This Section defines the general framework we use for modeling star-shaped tumor profiles defined by the normalized radial function $r_{t}(\phi)$. In Section 4.2 we will specify the model in more detail, adapted to specific tumor dynamics.

The basic notion is that of an independently scattered random measure (i.s.r.m) on continuous time-space, $\mathbb{R} \times \mathbb{R}$. These measures associate a random number with any subset of $\mathbb{R} \times \mathbb{R}$. Whenever two subsets are disjoint, the associated numbers are independent, and the measure of a disjoint union of sets almost certainly equals the sum of the measures of the individual sets. For a mathematically more rigorous definition of i.s.r.m.'s and their theory of integration, see $[7,9,10]$.

Independently scattered random measures provide a natural basis for describing uncorrelated noise processes in space and time. A special class of i.s.r.m.'s is that of factorisable and homogeneous Lévy bases, where the distribution of the measure of each 
set is infinitely divisible and does not depend on the location of the subset. In this case, it is easy to handle integrals with respect to the Lévy basis using the well-known Lévy-Khintchine and Lévy-Ito representations for Lévy processes. Here, we state the result and point to [7] for greater detail and rigour.

Let $Z$ be a factorisable and homogeneous Lévy basis on $\mathbb{R} \times \mathbb{R}$ such that $Z(A)$ is infinitely divisible for any $A \subset \mathbb{R} \times \mathbb{R}$. Then we have the fundamental relation

$$
\mathrm{E}\left\{\exp \left\{\int_{A} h(a) Z(\mathrm{~d} a)\right\}\right\}=\exp \left\{\int_{A} \mathrm{~K}[h(a)] \mathrm{d} a\right\},
$$

where $h$ is any integrable deterministic function, and $\mathrm{K}$ denotes the cumulant function of $Z(\mathrm{~d} a)$, defined by

$$
\mathrm{K}[\xi] \mathrm{d} a=\ln \mathrm{E}\{\exp \{\xi Z(\mathrm{~d} a)\}\} .
$$

The usefulness of $(15)$ is obvious: it permits explicit calculation of the correlation function of the integrated and $h$-weighted noise field $Z(\mathrm{~d} a)$ once the cumulant function $\mathrm{K}[h(a)]$ is known.

In particular, we define a spatio-temporal radial process $r_{t}(\phi)$ on $\mathbb{R} \times[0,2 \pi]$ as

$$
r_{t}(\phi) \equiv \exp \left\{\int_{A_{t}(\phi)} h\left(t, \phi ; t^{\prime}, \phi^{\prime}\right) Z\left(\mathrm{~d} \phi^{\prime}, \mathrm{d} t^{\prime}\right)\right\}
$$

where the so-called ambit sets $A_{t}(\phi) \subset \mathbb{R} \times[0,2 \pi]$ are defined in a cyclic way.

We define general spatio-temporal correlators $c_{n_{1}, n_{2}}(\phi, t ; \Delta \phi, \Delta t)$ as

$$
c_{n_{1}, n_{2}}(\phi, t ; \Delta \phi, \Delta t) \equiv \frac{\mathrm{E}\left\{r_{t}(\phi)^{n_{1}} r_{t+\Delta t}(\phi+\Delta \phi)^{n_{2}}\right\}}{\mathrm{E}\left\{r_{t}(\phi)^{n_{1}}\right\} \mathrm{E}\left\{r_{t+\Delta t}(\phi+\Delta \phi)^{n_{2}}\right\}}
$$

The definition (18) of general spatio-temporal correlators coincides with the definition (4) for spatial correlators for $\Delta t=0$ and omitting the variable $\phi$ on the left hand side of (18) for a statistically isotropic process $r_{t}(\phi)$.

Using (15), we immediately get

$$
c_{n_{1}, n_{2}}(\phi, t ; \Delta \phi, \Delta t)=\exp \left\{\int_{A(t, \phi ; \Delta t, \Delta \phi)} \overline{\mathrm{K}}\left[\phi, t ; \Delta \phi, \Delta t ; \phi^{\prime}, t^{\prime}\right] \mathrm{d} t^{\prime} \mathrm{d} \phi^{\prime}\right\},
$$

where

$$
\begin{aligned}
\overline{\mathrm{K}}\left[\phi, t ; \Delta \phi, \Delta t ; \phi^{\prime}, t^{\prime}\right]= & \mathrm{K}\left[n_{1} h\left(t, \phi ; t^{\prime}, \phi^{\prime}\right)+n_{2} h\left(t+\Delta t, \phi+\Delta \phi ; t^{\prime}, \phi^{\prime}\right)\right] \\
& -\mathrm{K}\left[n_{1} h\left(t, \phi ; t^{\prime}, \phi^{\prime}\right)\right]-\mathrm{K}\left[n_{2} h\left(t+\Delta t, \phi+\Delta \phi ; t^{\prime}, \phi^{\prime}\right)\right]
\end{aligned}
$$

and

$$
A(t, \phi ; \Delta t, \Delta \phi)=A_{t}(\phi) \cap A_{t+\Delta t}(\phi+\Delta \phi)
$$

is the overlap of the ambit sets associated to the points $(t, \phi)$ and $(t+\Delta t, \phi+\Delta \phi)$. Similar relations hold for correlators involving more than two spatio-temporal positions. In the Lévy based framework (17), modelling of a certain correlation structure for the spatiotemporal process $r_{t}(\phi)$ reduces to specifying the background field $Z$, the weight-function $h$ and the ambit sets $A_{t}(\phi)$ such that (19) holds. These three degrees of freedom of the modelling framework (17) can be chosen arbitrarily and independently, which allows for modelling of a wide range of correlation structures. 


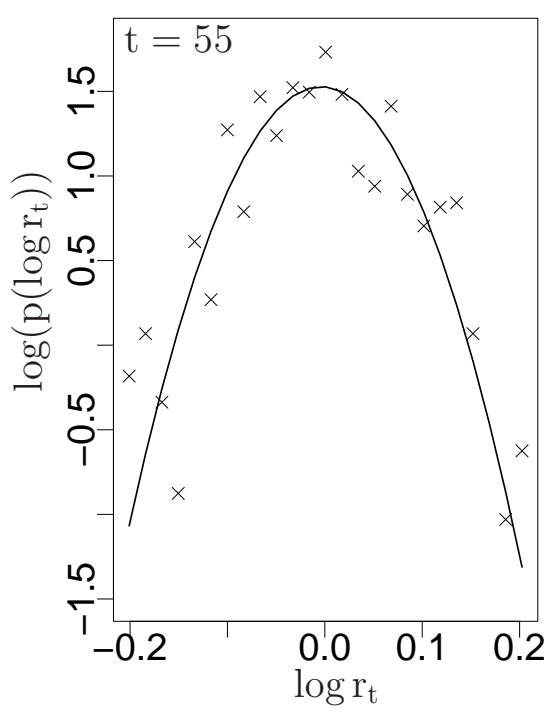

Figure 6: Density of $\log \left(r_{t}\right)$ at time $t=55$ (in hours). The solid line denotes the Gaussian approximation.

\subsection{The log-normal model}

The form of the spatial correlators (5) and the values of the self-scaling exponents (12) for the tumor profiles are the fundamental statistical properties that specify the structure of the general modelling framework (17) in more detail.

As the model for the dynamics of the normalized radii $r_{t}(\phi)$ of the star-shaped approximation of tumor profiles we propose

$$
\begin{gathered}
r_{t}(\phi)=\exp \left\{a(t) \int_{t-T(t)}^{t-t_{0}(t)} \int_{\phi-\pi}^{\phi+\pi} \cos \left(\phi-\phi^{\prime}\right) Z\left(\mathrm{~d} t^{\prime} \times \mathrm{d} \phi^{\prime}\right)\right. \\
\left.+h(t) \int_{t-t_{0}(t)}^{t} \int_{\phi-g_{t}\left(t^{\prime}\right)}^{\phi+g_{t}\left(t^{\prime}\right)} Z\left(\mathrm{~d} t^{\prime} \times \mathrm{d} \phi^{\prime}\right)\right\},
\end{gathered}
$$

where $Z$ is a normal Lévy basis, i.e. the random numbers $Z(\mathrm{~d} t \times \mathrm{d} \phi)$ are distributed according to a Gaussian law with mean $\mu \mathrm{d} t \mathrm{~d} \phi$ and variance $\sigma^{2} \mathrm{~d} t \mathrm{~d} \phi$. The assumption about the Gaussianity of $Z$ implies $\log \left(r_{t}(\phi)\right)$ to have a Gaussian distribution. Figure 6 shows the logarithm of the density of $\log \left(r_{t}\right)$ at time $t=55$. There is considerable scatter but the Gaussian distribution (solid line) seems to be a reasonable approximation. Similar results hold for all times $t$ that are displayed in Figure 1. Figure 7 shows the estimated mean $\mu_{t}$ and variance $\sigma_{t}^{2}$ of $\log r_{t}$ as a function of time. The normalization $\mathrm{E}\left\{r_{t}\right\}=1$ and the ansatz (22) implies $\sigma_{t}^{2} / 2+\mu_{t}=0$ which is confirmed by the estimated values of $\mu_{t}$ and $\sigma_{t}^{2}$ and can be seen as supporting the assumption of a Gaussian bachground field $Z$.

As we will show below, the first term on the right hand side of (22) is responsible for the validity of the cosine law (5) and the second term on the right hand side of (22) is associated with the deviations from the cosine law at small angular distances. We call the first term on the right hand side of (22) the large scale term and the second term of the right hand side of (22) the small scale term.

The ambit set associated with the large scale term is assumed to be a rectangular of the form $\left[t-T(t), t-t_{0}(t)\right] \times[\phi-\pi, \phi+\pi]$. The deterministic function $T(t)$ as 

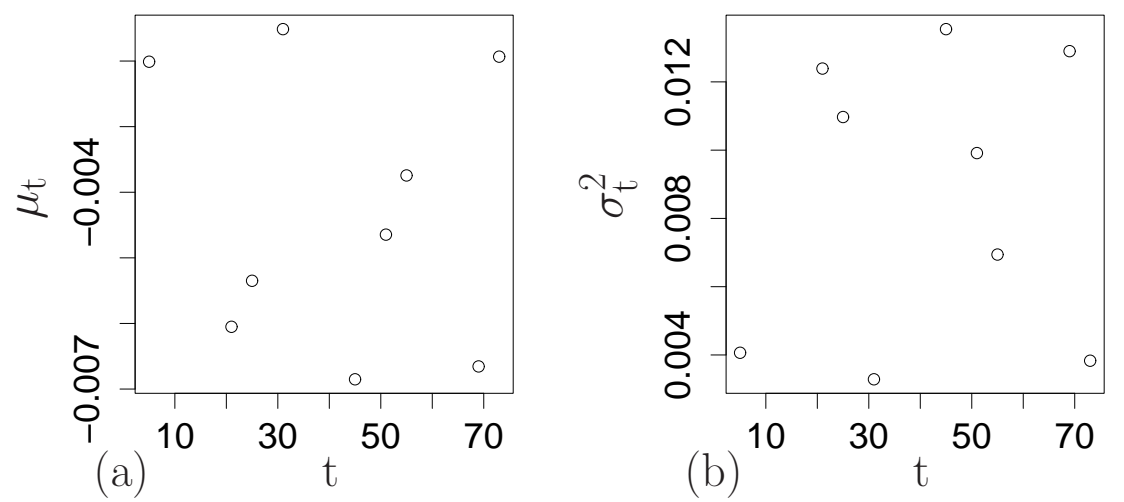

Figure 7: (a) Estimated mean $\mu_{t}$ and (b) estimated variance $\sigma_{t}^{2}$ at times $t=$ $5,21,25,31,45,51,55,69,73$ (in hours).

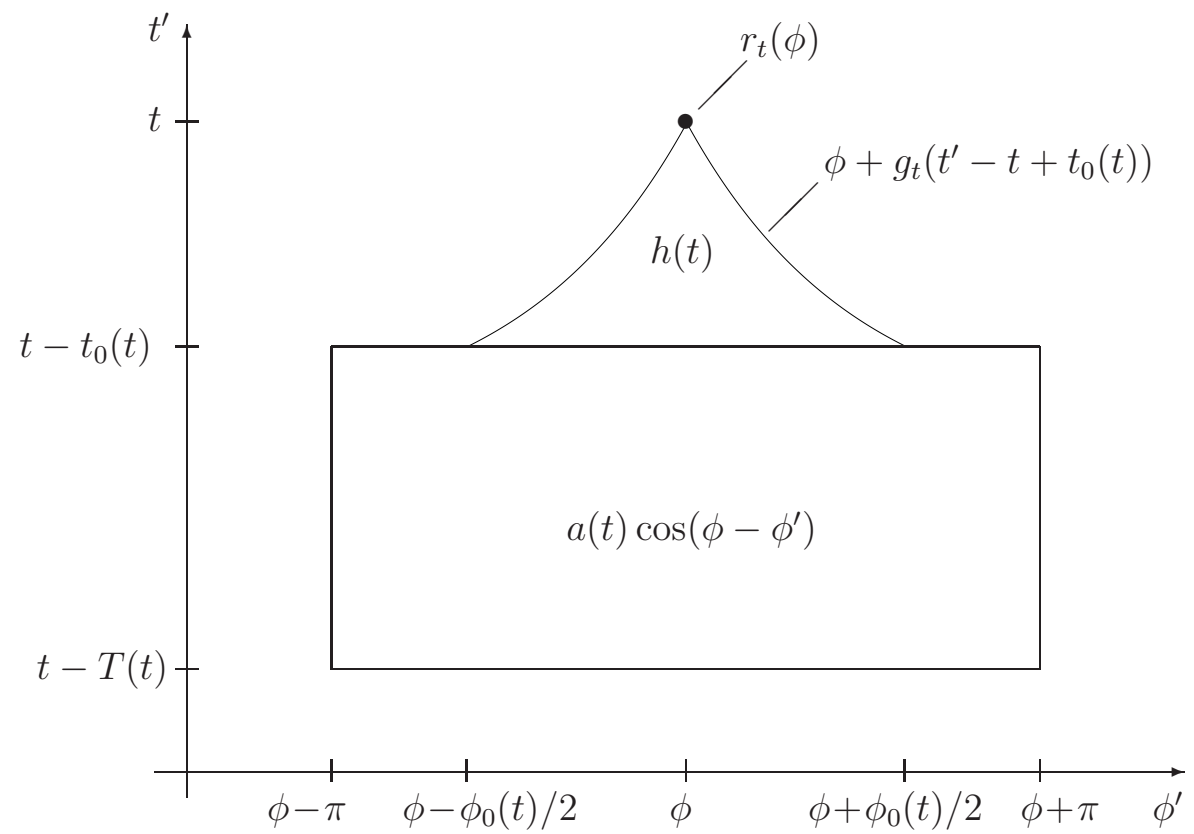

Figure 8: Illustration of the ambit set associated to $r_{t}(\phi)$ according to the ansatz $(22)$. The regions of different weight functions $h(t)$ and $a(t) \cos \left(\phi-\phi^{\prime}\right)$ are indicated. 
the maximum of the temporal extension of the large scale ambit set in the past can be interpreted as the decorrelation time of the radius process and $t_{0}(t)$ as the decorrelation time of the small scale term.

The ambit set associated with the small scale term is assumed to be determined by a deterministic and monotonically decreasing function $g_{t}$ defined on $\left[0, t_{0}(t)\right]$ and satisfying $g_{t}\left(t_{0}(t)\right)=0$. The small scale ambit set at $(\phi, t)$ is

$$
\left\{\left(\phi^{\prime}, t^{\prime}\right):-g_{t}\left(t^{\prime}-t+t_{0}(t)\right) \leq \phi^{\prime}-\phi \leq g_{t}\left(t^{\prime}-t+t_{0}(t)\right)\right\}
$$

with cyclic definition in the angle. The large scale term extends over the whole full circle. These two parts of the ambit set are weighted differently according to the deterministic functions $a(t) \cos \left(\phi-\phi^{\prime}\right)$ and $h(t)$ for the large scale term and the small scale term, respectively. Figure 8 illustrates the shape of the ambit set together with the corresponding weight functions.

The choice of the form of the ambit set and the weight function of the large scale term in (22) is motivated by the cosine law (5). Using (19) for $\Delta t=0$, it is straightforward to show that with the ansatz (22) the spatial correlators follow the observed cosine behaviour (5) with deviations at small scales, caused by the small scale term in (22)

$$
\ln \left(c_{n_{1}, n_{2}}(t, \Delta \phi)\right)=d_{n_{1}, n_{2}}(t) f_{t}(\Delta \phi) \mathbf{1}_{\left[0,2 g_{t}(0)\right]}(\Delta \phi)+b_{n_{1}, n_{2}}(t) \cos (\Delta \phi),
$$

where the large scale amplitude $b_{n_{1}, n_{2}}(t)$ is given by

$$
b_{n_{1}, n_{2}}(t)=n_{1} n_{2} a(t)^{2} \pi\left(T(t)-t_{0}(t)\right) .
$$

The small scale amplitude $d_{n_{1}, n_{2}}(t)$ has the form

$$
d_{n_{1}, n_{2}}(t)=n_{1} n_{2} h(t)^{2}
$$

and we set

$$
f_{t}(\Delta \phi)=V_{t}(\Delta \phi)=\int_{0}^{g_{t}^{(-1)}(\Delta \phi / 2)}\left(2 g_{t}(s)-\Delta \phi\right) \mathrm{d} s
$$

where $V_{t}(\Delta \phi)$ is the overlap of the ambit sets of the small scale terms separated by the angular distance $\Delta \phi$. Here, $g_{t}^{(-1)}$ denotes the Inverse of the bounding function $g_{t}$. The amplitude of the cosine law (5) is solely determined by the weight function $a(t)$ and the temporal extension $T(t)-t_{0}(t)$ of the large scale term. The deviations from the cosine law at scales $\Delta \phi<\phi_{0}(t)$ only depend on the weight function $h(t)$ and the overlap $V_{t}(\Delta \phi)$ of the small scale ambit sets. The critical angle $\phi_{0}(t)$ is given by

$$
\phi_{0}(t)=2 g_{t}(0)
$$

independent of the order $\left(n_{1}, n_{2}\right)$.

The modelling potential of the ansatz (22) for the dynamics of tumor profiles lies in the fact that the cosine behaviour at large scales can be modelled independently of the deviations at the small scales. In particular, a suitable choice of the bounding function $g_{t}(s)$ allows to model any monotonically decreasing overlap $V_{t}(\phi)$ and, consequently, any monotonically decreasing deviation $d_{n_{1}, n_{2}}(t) f_{t}(\Delta \phi)$.

The cosine law at the large scales is associated with the large scale ambit set, that correlates $r_{t}(\phi)$ with the radii at all angular positions. In contrast, the term causing the deviations at small angular distances is associated with the strongly localized small scale ambit set, correlating $r_{t}(\phi)$ with the radii inside a narrow angular domain $\left[\phi-\phi_{0}, \phi+\phi_{0}\right]$. This separation of scales involves the same Gaussian background field $Z$ which implies the order-dependent factor $n_{1} n_{2}$ for $b_{n_{1}, n_{2}}(t)$ and $d_{n_{1}, n_{2}}(t)$. As a consequence, the model (22) reproduces the empirical values of the self-scaling exponents (12). 


\subsection{Inference of the model parameters}

The model (22) for the normalized radii of the tumor profiles involves 7 parameters that can be chosen independently and arbitrarily.

The background normal Lévy basis $Z$ is characterized by the mean $\mu$ and the variance $\sigma^{2}$. We can always set $\sigma^{2}=1$ since any other choice can be absorbed in the definition of the weight functions $a(t)$ and $h(t)$. Thus we are left with 6 parameters, namely $\mu$, the weight functions $a(t)$ and $h(t)$, the decorrelation times $T(t)$ and $t_{0}(t)$ and the bounding function $g_{t}$, where we identify $2 g_{t}(0)=\phi_{0}(t)$. These parameters can all be inferred from spatio-temporal two-point correlators $(18)$ of orders $(1,1)$, using the isotropy of the profiles, and the law of $\log \left(r_{t}\right)$.

The logarithm of the normalized radius is distributed according to a Gaussian distribution with mean

$$
\mu_{t}=\mu h(t) V_{t}(0)
$$

and variance

$$
\sigma_{t}^{2}=\pi a(t)^{2}\left(T(t)-t_{0}(t)\right)+h(t)^{2} V_{t}(0) .
$$

Note that the mean $\mu_{t}$ only depends on the parameters of the small scale term.

From temporal correlators (18) with $\Delta \phi=0$ we infer the decorrelation time $T(t+\Delta t)$ as (see Figure 8)

$$
T(t+\Delta t)=\min \left\{\Delta t>0: \log \left(c_{1,1}(\phi, t, 0, \Delta t)\right)=0\right\} .
$$

The critical angle $\phi_{0}(t)=2 g_{t}(0)$ can be estimated from the small scale behaviour of spatial correlators (18) with $\Delta t=0$ as

$$
\phi_{0}(t)=2 g_{t}(0)=\min \left\{\Delta \phi>0: \log \left(c_{1,1}(\phi, t, \Delta \phi, 0)\right)-b_{1,1}(t) \cos (\Delta \phi)=0\right\},
$$

where $b_{1,1}(t)$ is inferred as the amplitude of the large scale cosine behaviour of spatial correlators.

Next, we assume

$$
\phi_{0}=\sup \left\{\phi_{0}(t): t>0\right\}<\pi,
$$

which is clearly true for the star-shaped tumor profiles (see Figure 2 and Figure 4(a)). Having in mind that for $\Delta \phi>\phi_{0}$ the small scale ambit sets do not contribute to the overlap of the ambit sets associated to space-time points $(\phi, t)$ and $(\phi+\Delta \phi, t+\Delta t)$, we get the temporal extension $t_{0}(t)$ of the small scale ambit set for $\Delta \phi>\phi_{0}$ as

$$
t_{0}(t)=T\left(t+T_{\min }\right)-T_{\min }
$$

where

$$
T_{\min }=\min \left\{\Delta t>0: \log \left(c_{1,1}(\phi, t, \Delta \phi, \Delta t)\right)=0\right\} .
$$

For the estimation of the bounding function $g_{t}$ we assume $V_{t}(\Delta \phi)$ to be differentiable with respect to $\Delta \phi$. We abbreviate the deviations from the cosine law, using (26) and (27), as

$$
Y(t, \Delta \phi)=d_{1,1}(t) f_{t}(\Delta \phi)=h^{2}(t) V_{t}(\Delta \phi) .
$$

Combining (36) for $\Delta \phi=0$ with (29) gives

$$
Y(t, \Delta \phi)=\frac{\mu^{2}(Y(t, 0))^{2}}{\mu_{t}^{2}} V_{t}(\Delta \phi)
$$


and, using (27), we finally get an expression for $g_{t}(s)=g_{t}(s, \mu)$, depending on $\mu$,

$$
g_{t}^{(-1)}(\Delta \phi / 2) \mu^{2}=\frac{\partial\left(\mu_{t}^{2} Y(t, \Delta \phi) /(Y(t, 0))^{2}\right)}{\partial \Delta \phi} .
$$

The relation (38) only depends on $\mu_{t}$ and $Y(t, \Delta \phi)$ that can be obtained from spatial correlators of order $(1,1)$. The mean $\mu$ of the background Lévy basis $Z$ can be found from the condition

$$
g_{t}^{(-1)}(0, \mu)=t_{0}(t)
$$

It remains to infer the weight functions $a(t)$ and $h(t)$. Using the estimates for $g_{t}$ and $\mu$ we may calculate the volume of the small scale ambit set (27) and from (29) we get

$$
h(t)=\frac{\mu_{t}}{\mu V_{t}(0)}
$$

The weight function $a(t)$ of the cosine term can easily be obtained from the estimated amplitude of the cosine law (5) as

$$
a(t)=\sqrt{\frac{b_{1,1}(t)}{\pi\left(T(t)-t_{0}(t)\right)}} .
$$

In summary all parameters of the dynamical model (22) can, in principal, be estimated from the law of $\log \left(r_{t}\right)$ and the spatio-temporal two point correlators of order $(1,1)$.

\section{Simulation}

The data set we analyze in this paper does not have the quality to allow to perform a proper estimation of the parameters of the dynamical model (22), as outlined in the previous Section. The temporal resolution of the data set is insufficient to infer the decorrelation times $T(t)$ and $t_{0}(t)$. Moreover, we only have access to one realisation of the tumor profile at a fixed time $t$. Therefore, the above outlined way to estimate the parameters of the small scale term of the dynamical model is not feasible.

To overcome these shortcomings associated with the quality of the data at hand, we restrict the simulations to a simplified version of the model (22). For the small scale ambit set we assume a triangular shape determined by

$$
g_{t}(s)=\frac{\phi_{0}(t)}{2}-\frac{\phi_{0}(t)}{2 t_{0}(t)} s, \quad s \in\left[0, t_{0}(t)\right]
$$

For the specification of the remaining model parameters we only use the estimated values of $\mu_{t}, \sigma_{t}^{2}$ and $b_{1,1}(t)$ and the form of the estimated spatial correlators $c_{1,1}(\Delta \phi)$. The parameters $\mu, T(t)$ and $t_{0}(t)$ are used as free parameters.

For the small scale deviations from the cosine behaviour (5) we get for the triangular small scale ambit set (42) the relation

$$
Y(t, \Delta \phi)=\frac{A(t)^{2}}{4 C(t)} \Delta \phi^{2}+A(t) \Delta \phi+C(t)
$$




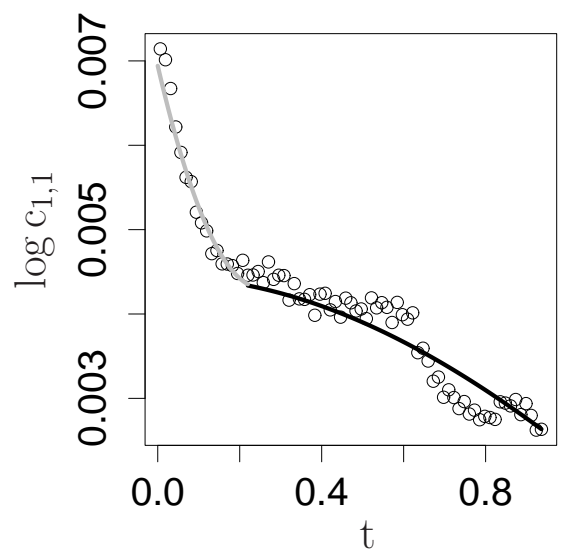

Figure 9: Approximation of the estimated spatial correlator $c_{1,1}(t, \Delta \phi)$ at time $t=55$ (in hours) within the model (22) with a triangular small scale ambit set. The gray line denotes the deviations from the cosine-law (44) and the black line denotes the cosinelaw (5).
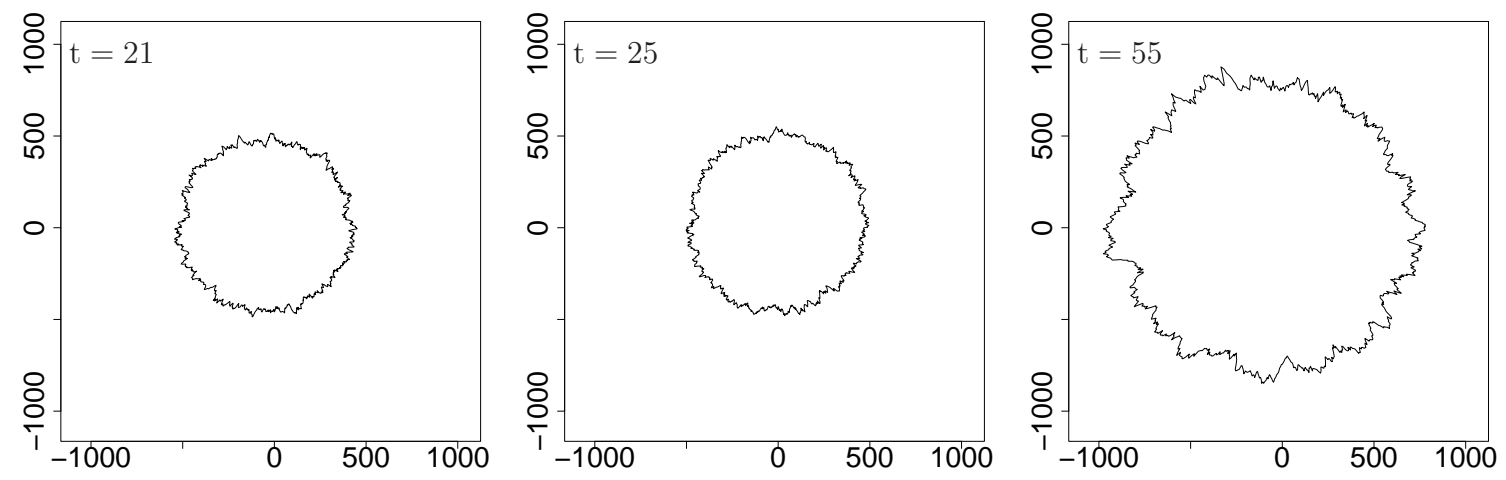

Figure 10: Simulation of the star-shaped tumor (arbitrary units) using the model (22) with a triangular small scale ambit set at times $t=21,25,55$ (in units of the finite step size $\Delta t$ ) with parameters $\mu=0.1$ and $\sigma^{2}=1$. The remaining parameters are listed in Table 1.

where $A(t)=h(t)^{2} t_{0}(t)$ and $C(t)=h(t)^{2} \phi_{0}(t) t_{0}(t) / 2$. The amplitude $C(t)$ can be rewritten using (30) and (41) as $C(t)=\sigma_{t}^{2}-b_{1,1}(t)$. Thus, the small scale deviations only depend on $A(t)$

$$
Y(t, \Delta \phi)=\frac{A(t)^{2}}{4\left(\sigma_{t}^{2}-b_{1,1}(t)\right)} \Delta \phi^{2}+A(t) \Delta \phi+\left(\sigma_{t}^{2}-b_{1,1}(t)\right) .
$$

We fit this form of the small scale deviation $Y(t, \Delta \phi)$ to the estimated spatial correlators for $\Delta \phi \leq \phi_{0}(t)$ and vary the critical angle $\phi_{0}(t)$ such that $Y(t, \Delta \phi) \mathbf{1}_{\left[0, \phi_{0}(t)\right]}+b_{1,1}(t) \cos \Delta \phi$ is continuous at $\Delta \phi=\phi_{0}(t)$. Figure 9 shows the results for $t=55$.

The parameters $a(t)$ and $h(t)$ are calculated using (41) and (40). Table 1 lists the estimated parameters of the model (22) with the triangular small scale ambit set for the times $t=21,25,55$.

For the simulations we discretize all integrals in (22) with a finite step size $\Delta t=1$ and an angular step size $2 \pi / N, N=1000$, which corresponds to the angular resolution in Figure 1. The free parameters $\mu, T(t)$ and $t_{0}(t)$ were tuned in a way such that the simulated profiles resemble the tumor profiles by visual inspection. 
Figure 10 shows the simulated tumor profiles at times $t=21,25,55$. The simulated profiles are reasonably similar to the tumor profiles in Figure 1. We obtain structures at very different scales comparable to the multi-scale structure in Figure 1. We restrict the simulations to times $t=21,25,55$ since the assumption of a triangular small scale ambit set and a constant mean $\mu$ for the background field $Z$ does not produce reasonable profiles for all times $t$. Therefore, the assumption of a triangular small scale ambit set should only be seen as an illustrating example.

Figure 11(a) shows the logarithm of the spatial correlators at time $t=55$ and the corresponding approximations $b_{n_{1}, n_{2}}(t) \cos \Delta \phi$. A comparison of Figure 11(a) with Figure $4(\mathrm{a})$ shows that the simulated amplitudes $b_{n_{1}, n_{2}}(t)$ differ from the corresponding estimated amplitudes. This difference may reflect the error in estimating the amplitudes $b_{n_{1}, n_{2}}(t)$.

Figures 11(b-d) show the self-scaling behaviour of the spatial correlators as estimated from the simulated data. The estimated slopes $k_{t}\left[m_{1}, m_{2} ; n_{1}, n_{2}\right]$ follow the relation (12) to a comparable accuracy as in Figure 5. The relation (12) can also be inspected from Figure 11(a) where the amplitudes $b_{n_{1}, n_{2}}(t)$ are approximately equidistant (see also Figure $4(\mathrm{a}))$.

The simulations show the ability of the modelling framework not only to reproduce the essentials of the correlation structure of tumor profiles, but also its potential to generate typical profiles. The overall appearance of the simualted profiles strongly depends on the form of the small scale ambit set. The assumption of a triangular ambit set is given as an example, a more detailed statement about the shape of the small scale ambit set can only be gained by analyzing larger data sets.

\section{Conclusion}

We presented a stochastic model for the dynamics of star-shaped planar tumor profiles. This type of modelling is embedded in a general Lévy based modelling framework, that allows to analytically control the spatio-temporal correlation structure. For tumor profiles, spatial correlators are mainly characterized by a cosine law at large scales with deviations at scales below a critical angle. Furthermore, correlators of different orders have the property of self-scaling. The observation of these striking statistical properties are an essential and original part of this paper. Assuming a Gaussian Lévy basis, the cosine law at large scales and the numerical values of the selfscaling exponents are intrinsic statistical properties of the proposed model. The deviations at small scales can be modelled independently and arbitrarily. With this degree of freedom, we believe that a higher quality of profile data will allow to model tumor profiles in greater detail.

The modelling framework (22) has been defined for the normalized radius $r_{t}(\phi)$. However, it equally applies to the modelling of the non-normalized radius $R_{t}(\phi)$. The definition of correlators is invariant under rescaling with the mean radius and the calculations in Section 4.3 and Section 5 do not use the normalization condition $\mathrm{E}\left\{r_{t}(\phi)\right\}=1$. Going from $r_{t}(\phi)$ to $R_{t}(\phi)$ is equivalent to replacing $h(t)$ with $h(t)-\log \left(\mathrm{E}\left\{R_{t}(\phi)\right\}\right) / V_{t}(0)$ (see (29)), keeping all other parameters unchanged.

In the present contribution we have dealt with tumor evolution in homogeneous media without specific anatomic constraints. Such homogeneous media can be taken to represent soft tissues, such as those in the brain. Inhomogeneous and non-isotropic media with specific boundary conditions can also be incorporated into the general modelling 

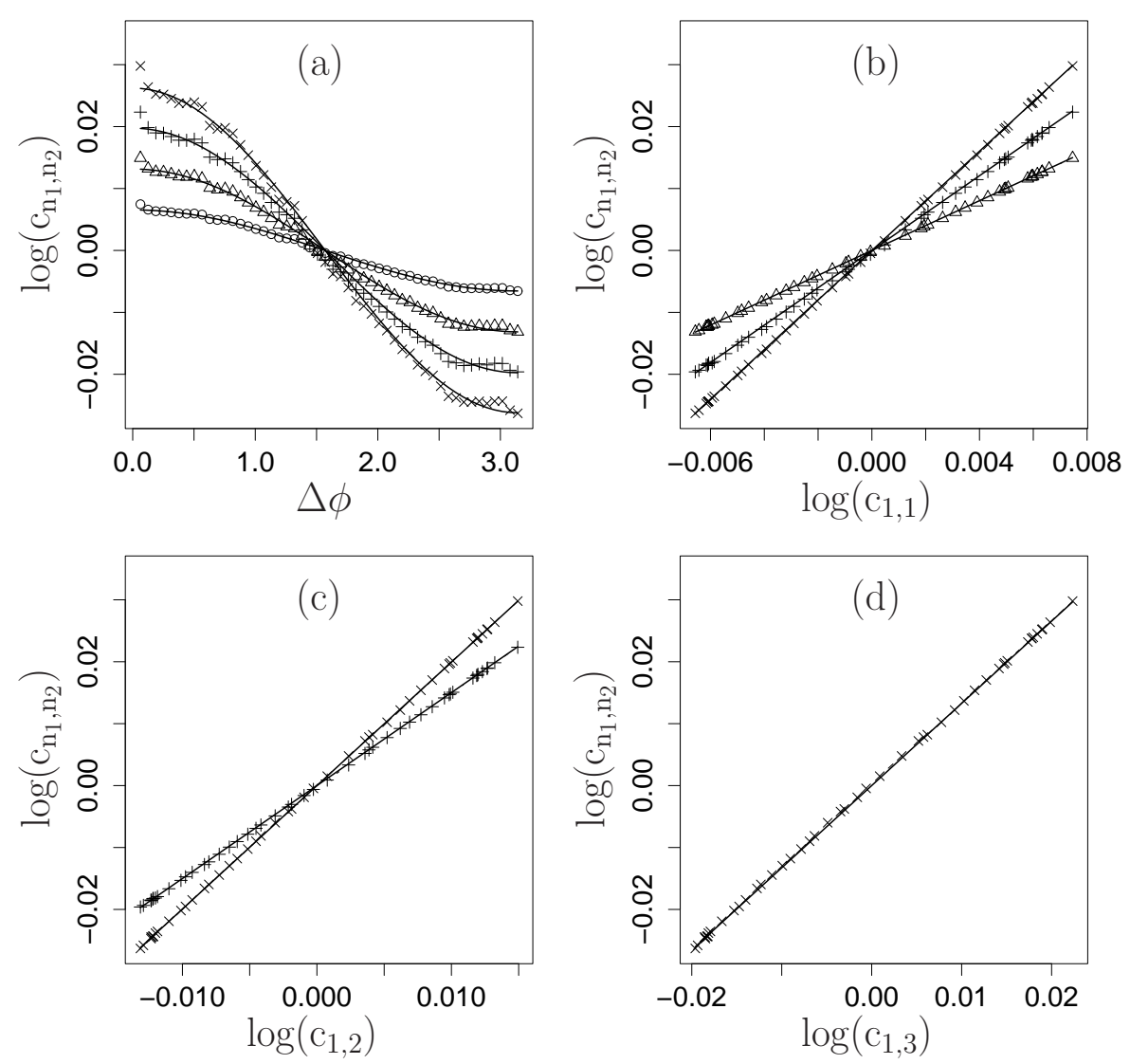

Figure 11: Logarithm of spatial correlators $c_{n_{1}, n_{2}}(t, \Delta \phi)$ of orders $\left(n_{1}, n_{2}\right)=(1,1)(\circ)$, $\left(n_{1}, n_{2}\right)=(1,2)(\triangle),\left(n_{1}, n_{2}\right)=(1,3)(+)$ and $\left(n_{1}, n_{2}\right)=(2,2)(\times)$ at time $t=55$ (in units of the finite step size $\Delta t$ ) as a function of $\Delta \phi$ (radiants) for the simulated data in Figure 10. The solid lines are of the form $b_{n_{1}, n_{2}}(t) \cos (\Delta \phi)$. (b) Logarithm of spatial correlators $c_{n_{1}, n_{2}}(t, \Delta \phi)$ as a function of $\log c_{1,1}(t, \Delta \phi)$ for $\left(n_{1}, n_{2}\right)=(1,2),(1,3),(2,2)$. The solid lines are of the form $k_{t}\left[1,1 ; n_{1}, n_{2}\right] \log c_{1,1}(t, \Delta \phi)$. (c) Logarithm of spatial correlators $c_{n_{1}, n_{2}}(t, \Delta \phi)$ as a function of $\log c_{1,2}(t, \Delta \phi)$ for $\left(n_{1}, n_{2}\right)=(1,3),(2,2)$. The solid lines are of the form $k_{t}\left[1,2 ; n_{1}, n_{2}\right] \log c_{1,2}(t, \Delta \phi)$. (d) Logarithm of spatial correlators $c_{2,2}(t, \Delta \phi)$ as a function of $\log c_{1,3}(t, \Delta \phi)$. The solid line is of the form $k_{t}\left[1,3 ; n_{1}, n_{2}\right] \log c_{1,3}(t, \Delta \phi)$. 
framework (17). For instance, identifying $r_{t}(\phi)$ in (17) with the non-normalized radius $R_{t}(\phi)$ and choosing the weight-function $h\left(t, \phi, t^{\prime}, \phi^{\prime}\right)$ to be localized around $\phi=\phi_{0}$ will result in growth that is predominantly in direction $\phi_{0}$.

For the application to characterize different types of tumors and the grading of the malignancy within one type of tumor, we believe that the analysis of the correlation structure in terms of spatio-temporal correlators may yield useful criteria for diagnosis purposes. In particular, the deviations of spatial correlators from the cosine law at small scales are strongly related to the roughness of the profiles at small scales and as such reflect the malignancy and the potential of the tumor being infiltrative [5].

\section{References}

[1] R. Peirolo and M. Scalerandi, Phys. Rev. E 70 (2004) 011902.

[2] A. Brú, J.M. Pastor, I. Fernaud, I. Brú, S. Melle and C. Berenguer, Phys. Rev. Lett. 81 (1998) 4008.

[3] P.P. Delsanto, A. Romano, M. Scalerandi and G.P. Pescarmona, Phys. Rev. E 62 (2000) 2547.

[4] M. Scalerandi, A. Romano, G.P. Pescarmona, P.P. Delsanto and C.A. Condat, Phys. Rev. E 59 (1999) 2206.

[5] W.B. Spillman, Jr., J.L. Robertson, W.R. Huckle, B.S. Govindan and K.E. Meissner, Phys. Rev. E 70 (2004) 061911.

[6] B. Capogrosso Sansone, P.P. Delsanto, M. Magnano and M. Scalerandi, Phys. Rev. E 64 (2001) 021903.

[7] O.E. Barndorff-Nielsen and J. Schmiegel, Usp. Mat. Nauk 159 (2004) 63.

[8] J. Schmiegel, Phys. Lett. A 337 (2005) 342.

[9] O. Kallenberg, Random Measures, (Akademie Verlag, Berlin, 1989).

[10] S. Kwapien and W.A. Woyczynski, Random Series and Stochastic Integrals: Single and Multiple, (Birkhäuser, Basel, 1992). 